\title{
Marivaux journaliste: point de vue et opinion publique
}

Jean-Pierre Montier

\section{(e) OpenEdition}

1 Journals

\section{Édition électronique}

URL : http://journals.openedition.org/studifrancesi/9087

DOI : 10.4000/studifrancesi.9087

ISSN : 2421-5856

Éditeur

Rosenberg \& Sellier

\section{Édition imprimée}

Date de publication : 1 juin 2008

Pagination : 19-40

ISSN : 0039-2944

\section{Référence électronique}

Jean-Pierre Montier, « Marivaux journaliste: point de vue et opinion publique », Studi Francesi [En ligne], 154 (LII | I) | 2008, mis en ligne le 30 novembre 2015, consulté le 13 janvier 2021. URL : http:// journals.openedition.org/studifrancesi/9087 ; DOI : https://doi.org/10.4000/studifrancesi.9087

\section{(c) (i)}

Studi Francesi è distribuita con Licenza Creative Commons Attribuzione - Non commerciale - Non opere derivate 4.0 Internazionale. 


\section{Marivaux journaliste: point de vue et opinion publique}

Le 22 mars 1722, Marie Anne Victoire, la jeune Infante d'Espagne, arrive à Paris. Le 9 janvier, sur les rives de la Bidassoa, elle avait été présentée au jeune Louis XV, dont le sacre est prévu pour octobre. Un an plus tôt, il avait été conclu que l'alliance entre la France et l'Espagne, mettant fin à la guerre commencée en 1717, serait scellée par une union conjugale. Le futur roi n'a que treize ans, la princesse espagnole en a quatre $^{1}$. Qu'importe la disconvenance des âges; les princes ne sont pas faits pour avoir une vie privée mais pour marier des dynasties, unir des États. Un mariage est une promesse de paix durable. Les peuples le savent, et les Parisiens se rendent en foule au spectacle de l'arrivée de l'Infante, les rues de Paris étant fastueusement décorées. Dans le «Journal historique», Barbier rapporte:

Il y a un arc de triomphe à l'endroit de l'ancienne porte Saint-Jacques, au-dessus des Jacobins, avec cette inscription: Felici Adventui Lutetix; un autre attaché au Châtelet avec ces mots: Venit Expectata Dies; un au bout du pont Notre-Dame, et un dans la rue de la Ferronnerie avec plusieurs inscriptions [...]. Ce sont des toiles peintes avec un peu d'or, et cela fait assez bien².

Le chroniqueur s'acquitte de sa tâche, donne les détails qui firent cette journée mémorable. Pour cela, l'on orna Paris, l'on utilisa les perspectives et les monuments. Par la suite, l'on diffusa des représentations de l'événement. Une gravure anonyme figura la rencontre entre les deux enfants royaux ${ }^{3}$.

Moins de trois semaines après ces solennités, le 10 avril 1722, était approuvée la publication de la cinquième feuille du «Spectateur français» de Marivaux. De mai 1721 jusqu'en octobre 1724, il publie vingt-cinq «feuilles volantes», selon ses termes, qui sans relever tout à fait du journalisme n'en adoptent pas moins le principe d'une parution (presque) régulière. Marivaux s'inspire d'ailleurs du titre du premier quotidien anglais, le «Spectator», fondé par Addison et Steele en 1698. La date de la mise en vente n'est pas connue; quelques jours plus tard, sans doute. Au prix de six sols, les Parisiens peuvent alors trouver chez quelques libraires, où ils achètent aussi le «Mercure» (Cavelier, au Palais, Cavelier fils, rue Saint-Jacques, Le Breton, rue Guénégaud, Pissot, quai des Augustins), la relation de l'arrivée de l'Infante.

À l'exception des premières lignes, il consacre à ces réjouissances politiques et populaires la totalité de la cinquième feuille, soit une quinzaine de pages imprimées ${ }^{4}$.

(1) Elle passera trois années à Paris, avant d'être renvoyée à Madrid, Louis XV épousant finalement Marie Leczinska en 1725.

(2) Voir Marivaux, Journaux et Euvres diverses, édition Deloffre et Gilot, Paris, Bordas, 1988 («Classiques Garnier»), note 84, p. 585. Ce sera notre édition de référence.

(3) «La réception de l'Infante», conservée à la
Bibliothèque nationale, est reproduite sur la planche $n^{\circ} 8$ de l'édition citée. Il existe aussi un tableau de Nicolas Largillière resté à l'état d'esquisse, qui était destiné à l'Hôtel de Ville de Paris (Allégorie des fiançailles de Louis XV avec l'Infante), fut peint en 1722, et est aujourd'hui conservé au Musée Carnavalet.

(4) Les feuilles évoquées par Marivaux sont des 
Pour un auteur qui ne cesse de clamer qu'il n'en est pas un et qu'il ne fait qu'aller par monts et par vaux, au fil d'une plume badine et libertine, Marivaux, on va le voir, manie ensemble finesse et géométrie. Il va procéder à une série de contre-champs qui sont autant de dénis de représentation de ce qu'il prétend pourtant donner à voir.

Première échappatoire: à la fin de la quatrième Feuille ${ }^{5}$, le rédacteur avait promis de raconter un rêve consacré à l'amour. Ici, il prie son «Ami lecteur» d'accepter qu'il change de sujet, évoquant son humeur (son «libertinage d'idées»): l'amour peut attendre, place à l'actualité ${ }^{6}$ ! En effet, les festivités à Paris ont dû impressionner, être l'objet de toutes les conversations: Marivaux n'a aucune peine à justifier qu'il se plie à l'impérieuse nécessité de se raccorder au présent immédiat:

Je viens de voir l'entrée de l'Infante. J'ai voulu parcourir les rues pleines de monde, c'est une fête délicieuse pour un misanthrope que le spectacle d'un si grand nombre d'hommes assemblés; c'est le temps de sa récolte d'idées?

D'entrée, exit le spectacle, au profit des «idées», c'est-à-dire d'un tout autre type de représentation. L'objet des délices de son Spectateur n'est pas exactement ce qui attire les badauds. Marivaux, on s'en doute déjà, ne nous décrira rien d'un fait public dont à l'évidence la signification politique et historique même tiennent pourtant au «muthos» plus qu'au «logos»: la solennité de la présentation des enfants doit ancrer dans les mentalités, mieux que la signature d'un traité, le concret de la Paix. La phrase pivot «Je viens de voir l'entrée de l'Infante» introduit le sujet principal, feint de proposer au lecteur de satisfaire son intérêt, joue de la publicité donnée à l'événement. Mais dès l'attente posée, l'horizon visuel du texte bascule diamétralement. Si Marivaux se présente comme «témoin» c'est pour mieux écarter la piste descriptive: l'entrée de l'Infante, ce sont des rues pleines de monde, donc autant de difficultés à percevoir qu'à circuler. Pour voir quoi? Fort peu de choses en réalité... Il s'agit à la fois d'un problème littéraire et philosophique. Littérairement, c'est la question du descriptif, à propos d'un fait foisonnant et confus. Stendhal le traitera plus tard, dans le célèbre épisode de la bataille de Waterloo «vue» par Fabrice. En ce sens, que le spectateur ne voie rien de l'événement est un trait de réalisme. Philosophiquement, c'est la question de la nature et de la fonction du spectacle qui se posent. Le véritable spectacle, c'est le peuple qui le donne, inconsciemment sans doute, mais cette ignorance même est un trait caractéristique du fonctionnement inaperçu des institutions politiques. On sent poindre Pascal. Si Marivaux ne nie pas l'intérêt du spectacle (qui est «curieux» écrit-il plus loin), son ambition va être d'en penser le fondement, d'en démonter le mécanisme. Pour cela, il va l'envisager du côté de la «pensée de derrière», dégager la «raison des effets», selon le vocabulaire pascalien. Mais seulement le vocabulaire, car,

feuilles d'imprimerie. Pliées en huit folios, elles offraient seize pages, la première étant réservée au titre et déclinant le prix, le privilège, les noms et adresses des libraires.

(5) La date de l'approbation de la Quatrième feuille du Spectateur français est le 28 février 1722. Elle fut en vente au début de mars, mais l'on constate qu'il s'écoula un mois avant que ne sorte la cinquième. En réalité, la régularité de parution des trois journaux de Marivaux (L'Indigent philosophe et Le Cabinet du Philosophe succédèrent au Spectateur) ne fut jamais possible.

(6) Le mot «actualité» est évidemment un anachronisme. Je l'emploie toutefois par souci heuristique: si Marivaux ne dispose pas des moyens pour publier ses feuilles au fil des nouvelles ou des informations, et s'il n'en forme d'ailleurs pas non plus le projet, en revanche il anticipe, comme on verra, des rapports très modernes à l'événement. Ainsi, le «libertinage d'idées» expliquant le report du rêve n'est-il pas seulement une marque de ton littéraire, mais sans doute aussi une posture d'improvisation, comme s'il avait dû changer la une à chaud! Situation fictive, bien entendu, mais créant un rapport de connivence inédit avec le lecteur, ce qui intéresse MarivauX en tant qu'il est partie prenante dans la querelle des Anciens et des Modernes, du côté des seconds.

(7) Marivaux, «Le Spectateur français», in Journaux et Euvres diverses, cit., p. 132. 
on va le voir, Marivaux n'est pas pascalien jusqu'au bout. N'importe: les véritables acteurs de la constitution du sens politique sont bien dans les rues. C'est ce qui justifie l'adoption de la vue diamétralement opposée à la scène. En effet, si l'acte politique fonctionne pleinement selon les règles du spectacle et y gagne en majesté, alors il conviendra en retour que l'on prenne soin de vérifier comment le public, comme au théâtre au fond, y est aussi maître d'exprimer ses acquiescements ou ses déplaisirs, tout fasciné qu'il soit supposé être...

À la suite de ce préambule, succinct mais gros d'enjeux fondamentaux, le texte de la cinquième feuille va se déployer en plusieurs moments enchâssés, qui tous auront pour point commun d'éluder derechef la vision de l'événement pour mieux envisager les conditions éthiques et politiques du spectaculaire. La dimension théâtrale étant le modèle générique qui permettra à Marivaux d'isoler des moments successifs dans la profusion tous azimuts des vues possibles, nous avons choisi de dénommer ces phases «scènes», et de les numéroter de 1 à 4.

\section{Scène 1: Le Savetier qui ne voulait rien voir}

Le Spectateur est d'abord tiré de ses réflexions par la vue d'un «pauvre savetier» qui travaille dans sa boutique. Quoiqu'il y soit comme aux premières loges de ce théâtre en pleine rue, il demeure indifférent à l'objet des passions de la foule, ostensiblement concentré sur son travail, critiquant la curiosité bruyante et sans motif des badauds. Premier objet d'attention, l'incuriosité: le Spectateur entreprend de «voir de près ce philosophe subalterne et d'examiner quelle forme pouvaient prendre des idées philosophiques dans la tête d'un homme qui raccommode des souliers.» Le voilà chez le savetier, qui lui accorde «asile dans sa boutique contre la foule», et à qui il demande: «Comment! [...] vous travaillez, pendant que vous pouvez voir de si belles choses, mon bon homme!» Nous sommes en réalité dans une parodie d'une fable de La Fontaine, Le savetier et le financier (Livre VIII, 2). Le fabuliste en avait fait un modèle de la sagesse épicurienne incarnée dans un personnage populaire: d'abord berné par le financier insomniaque qui lui achète contre de l'or son silence (il chantait en travaillant!), le savetier finit par lui rendre sa bourse en échange de sa joie de vivre retrouvée. Le registre parodique est souligné par Marivaux quelques lignes plus loin: après le départ du Spectateur, son savetier lui aussi se met à chanter:

La scène a fini par une petite chanson qu'il a entonnée; ma curiosité satisfaite, je me suis retiré de sa boutique pour aller butiner quelque nouveauté ailleurs.

Déjà, chez La Fontaine, le récit comportait une dimension morale et économique: le savetier est l'artisan opposé à l'homme de finances qui prospère sans rien faire, sinon utiliser la capacité de l'argent à fructifier. Le premier ne compte ni ses heures ni son or (qu'il se garde d'amasser: il est dans la subsistance), au contraire du second que la spéculation enrichit immoralement. La nature même de sa richesse fait de lui un oisif, donc un inquiet et un malheureux. Emprunté à l'univers du fabuliste, le savetier de Marivaux va donc fournir le point de vue de ce qu'on pourrait appeler intempestivement «le monde du travail», et justifier implicitement plusieurs questions. Comment est-il concevable de perdre son temps à un tel spectacle? Quelle valeur sociale peut avoir un événement qui n'est destiné qu'à ceux qui ne travaillent pas, qu'il s'agisse de ceux qui le donnent (à quel prix?) ou de ceux qui le regardent? Que cache cette complicité momentanée entre oisifs, qu'ils soient Princes ou bien spectateurs? En quelle (fausse?) monnaie ce plaisir se paie-t-il?

Que cet épisode au réalisme problématique serve de prétexte à de telles ques- 
tions est naturellement contenu dans la parodie même: le lecteur est averti qu'il s'agit moins de rapporter des observations attestées que de se situer dans une sorte de chronique ludique surinvestie de littérature. Si la forme du «reportage» sert de modèle, la pression de l'intertexte littéraire la concurrence, sans toutefois complètement la récuser.

Pardi! m'a-t-il répondu, Monsieur, cela est trop beau pour de petites gens comme nous; cela ne nous appartient pas de voir ces beautés-là; cela est bon pour vous autres gens qui avez votre pain cuit, et qui avez le temps de mettre votre journée à rien faire.[...] Voilà quatre escabeaux dans ma boutique; je suis content comme un roi, avec cela et mes savates; je m'en accommode à merveille, quand je ne m'amuse pas à regarder toutes ces braveries-là; mais sitôt que je vois tant de beaux équipages et tout ce monde qu'il y a dedans, mes escabeaux et mes savates me fâchent; je deviens triste; je n'ai plus de cœur à l'ouvrage.

Le premier «point de vue» (paradoxal, puisqu'il s'agit d'un refus de rien voir) recueilli par le Spectateur frappe par sa radicalité. Par-delà le registre supposé bas dans lequel s'exprime le personnage de «brut Socrate» qui amuse son auditeur ( «J'interrompis ce discours d'un sourire»), son refus de prendre part au jeu social que constitue le spectacle se justifie par la mise en évidence des conditions économiques qui rendent celui-ci possible. Quand le Savetier quitte des yeux son labeur et qu'il se met à considérer l'usage fait des richesses pour des futilités, il perd le plaisir qu'il prend à travailler! La prospérité et le pouvoir, qui sont fondamentalement le produit du travail et de la vie pratique, dès lors qu'ils s'exhibent comme admirables réifient ceux qui en sont les causes initiales, c'est-à-dire les gens du peuple, dans la double acception économique et juridique du terme. Cette manipulation semble invalider toute fonction positive du spectacle, et paraît anticiper Guy Debord lorsqu'il écrit:

Le spectacle se représente à la fois comme la société même, comme une partie de la société, et comme instrument d'unification. En tant que partie de la société, il est expressément le secteur qui concentre tout regard et toute conscience. Du fait même que ce secteur est séparé, il est le lieu du regard abusé et de la fausse conscience; et l'unification qu'il accomplit n'est rien d'autre qu'un langage officiel de la séparation généralisée 8

Mais, une fois le problème posé dans toute sa force, la finesse de Marivaux va consister à ne pas s'en tenir à cette radicalité première. «Je ne lui ai rien répondu, sinon qu'il avait raison», note le Spectateur. Etait-ce inutile d'argumenter avec le «philosophe subalterne» du fait qu'il aurait été inaccessible à la contradiction? Étaitce qu'il fallait sans répliquer se ranger aux évidences qu'il assénait péremptoirement? Est-il possible de dialoguer avec le peuple? Le texte, à ce moment de son développement, laisse ouvert le choix de savoir s'il faut acquiescer aux dires du peuple pour éviter de le braquer, ou bien s'il convient, quelque naïves qu'elles puissent paraître, de supposer des «opinions du peuple saines», qu'il faudra aller trouver à un autre niveau d'analyse. Alors se présentera, comme on verra plus loin, la question: de quoi est faite l'opinion publique, si elle n'est ni l'addition de celle des individus, y compris les plus «bruts», ni leur constitution par un point de vue éclairé qui subsumerait leurs différences et leur multiplicité? En attendant, son Spectateur va continuer de butiner...

Remarquons cependant, et sans que l'on puisse affirmer que Marivaux se parodie aussi lui-même, que son savetier est un avatar du personnage d'Arlequin, du moins celui de la pièce qu'il fera représenter un an plus tard, en avril 1723, La double

(8) G. Debord, La société du spectacle, troisième édition, Paris, Gallimard, 1992 («Folio»), p. 16. 
inconstance. Arlequin y répond à Trivelin: «Alerte, alerte, paresseux, laissez vos chevaux à tant d'honnêtes laboureurs qui n'en ont point, cela nous fera du pain; vous marcherez et vous n'aurez pas les gouttes» $(I, 4)$. Au grand dam de Trivelin, qui est le serviteur servile, Arlequin, aussi libre et brut que le Savetier, critique les faux-semblants du pouvoir et ne se soucie «ni d'honneurs, ni de richesses, ni de belles maisons, ni de magnificence, ni de crédit, ni d'équipages.» Cet Arlequin, au costume bigarré, qui devant les serviteurs oisifs de la cour se demande «à quoi servent ces grands drôles bariolés qui nous accompagnent partout» $(I, 9)$ et qui, selon Silvia, «n'est pas plus gros monsieur que je suis grosse dame» $(\mathrm{I}, 1)$, présente des similitudes frappantes avec le savetier de la cinquième feuille, lequel serait en somme un mixte de personnage de fable et d'Arlequin transposé à la ville. Ils ont en commun le parler cru, les mots qui frappent par leur justesse, la lucidité à l'épreuve des artifices que les grands placent devant les yeux des humbles pour les fasciner. Plus fort que cet Arlequin qui, en 1720, était finalement «poli par l'amour», celui de La double Inconstance, contemporain du Savetier, est apte à tenir tête au Prince même:

Eh! Monseigneur, ne vous fiez pas à ces gens qui vous disent que vous avez raison avec moi, car ils vous trompent.[...] Sans ces gens-là, vous ne me chercheriez point chicane, vous ne diriez pas que je vous manque de respect parce que je vous représente mon bon droit: allez, vous êtes mon prince et je vous aime bien; mais je suis votre sujet, et cela mérite quelque chose. (III, 5)

Dans cette scène d'une violence contenue, les raisons exposées par Arlequin - auquel le Prince ne peut répondre que: «Vas, tu me désespères!» - sont indiscutablement légitimes. Au sens juridique: il en appelle au droit fondamental du royaume. Peu auparavant, lors d'un face-à-face burlesque avec le Seigneur (figure lui aussi de la servilité, celle des Grands, dont Saint-Simon sut dire aussi qu'ils pouvaient être moins que valets), Arlequin rappelait déjà au respect de l'impératif fondateur selon lequel «Noblesse oblige». Au Seigneur qui prétendait acheter sa complaisance en lui offrant des lettres de noblesse, Arlequin rétorque: «Ma noblesse n'oblige-t-elle à rien? car il faut faire son devoir dans une charge.» (III, 4).

Obligation; devoir: la suite du texte de Marivaux va précisément se porter en quête de ce que sont les devoirs qui justifient la mise en spectacle du pouvoir. Comment s'exercent-ils, à quels impératifs le Prince doit se plier pour que le peuple ne soit pas la dupe mais l'acteur du jeu politique? Nous retrouverons dans la scène 3 des échos de ces questions, adressées directement au Roi. Bien plus profond que Debord, qui ne voit dans le spectacle qu'un mécanisme unidirectionnel, manichéen, Marivaux va peu à peu poser les termes d'une économie du spectaculaire, penser ce qu'est un point de vue légitime, exposer les termes d'un jeu de rôles entre prince et sujets, base effective du commerce entre les hommes.

\section{Scène 2: Le peuple esbaudi ou l'empire de l'imaginaire}

Pourtant, rien n'est gagné d'avance! Lorsque le Spectateur a quitté la boutique du Savetier, il retrouve la foule, le peuple, admiratif et épaté, descendu dans la rue.

Je me suis amusé quelque temps de la populace qui se renversait la tête pour considérer les arcs de triomphe; et dans sa façon de voir, j'ai cru démêler que l'admiration du peuple pour une belle chose ne vient pas précisément de ce qu'elle est belle, mais bien des événements plus ou moins importants qui font qu'elle est exposée là, et qui la vantent à son imagination. 
Posons d'abord que le terme «populace», évidemment péjoratif, n'est pas nécessairement contradictoire avec l'idée développée plus loin d'un peuple qui pense et produit des opinions valables'. Marivaux n'est pas méprisant. Il est à la recherche d'hommes élémentaires, aptes à lui fournir le modèle servant à penser le phénomène auquel il s'intéresse: comment fonctionne l'imagination, que valent les appréciations qu'elle engendre? Percevoir, c'est imaginer et penser, former une perception qui va convoquer un jugement de type esthétique et politique, car il n'y a pas si loin d'un spectacle donné dans l'enceinte d'un théâtre à l'entrée solennelle d'un monarque dans une ville, comme il s'en pratiquait couramment depuis le Moyen-Âge. La populace n'est jamais qu'un parterre (et Molière n'avait-il pas fait l'éloge du parterre?), au jugement duquel, quoi que puissent en penser les tenants du mépris, l'observateur politique aussi bien que l'homme de théâtre se doivent de se régler. L'un et l'autre savent que si les villes ont des portes, des perspectives, des statues et des arcs, c'est qu'elles sont des scènes de la sociabilité, un théâtre de la vie en commun. Or, l'usage de l'imagination en effet y pose débat.

Tout ce passage, s'il se donne encore comme un reportage, ou une «chose vue», pour employer un terme un peu moins anachronique, va de nouveau être une plongée dans la littérature morale, sous l'égide non plus de La Fontaine, mais encore de Pascal.

Lecteur passionné et fervent admirateur de Pascal $^{10}$, Marivaux initie un mouvement dialectique fondé tout entier sur la transposition des divers niveaux d'interprétation tels qu'ils sont implicitement posés dans les Pensées. Le Savetier grincheux et pourfendeur du spectacle n'était jamais qu'un demi habile: il faut considérer le comportement du peuple, dénommé dans un premier temps «populace», pour comprendre ce qu'il en est au juste de la «raison des effets». Et de cette véritable «méditation pascalienne» ${ }^{11}$ pratiquée sur le vif de l'événement, émergera la question cruciale: le peuple est-il vraiment dupe? S'il accepte de l'être, est-ce pour autant qu'il suspend son jugement de manière définitive?

Dans cette seconde «scène» de la feuille $V$, en dépit de l'apparente incohérence, en réalité tout se tient grâce à la métaphore filée du tableau, celui-là même d'ailleurs que Marivaux va constamment se refuser à donner! Si voir est un acte d'intellection, alors qu'est-ce qui s'y donne à entendre? C'est pourquoi le Spectateur témoigne de ce qu'il écoute le peuple dire, et qui explicite presque phénoménologiquement ce qu'il voit:

J'entendais dire de tous côtés: Oh! que cela est beau! Et moi qui allais au principe de cette exclamation dans l'esprit du peuple, je la mettais en forme; et voici l'espèce d'argument qu'elle me rendait: Hé! vois-tu tout ce monde? c'est que l'Infante arrive. Tout ce que nous voyons là est fait pour elle; regardons bien, car assurément cela doit être beau. Oh! que cela est beau!

(9) Comme on le verra plus loin, le terme de «populace» est récurrent au moins jusqu'à la fin du siècle, y compris chez ceux des philosophes qui s'efforcent de penser la constitution de l'opinion publique, tel Condorcet même, dans la mesure où se pose justement le problème de l'articulation entre un peuple mal ou peu instruit et des «lumières» que viendraient lui dispenser les gens éclairés. Marivaux, quant à lui, ne fait pas la distinction entre le peuple et les instruits, supposant à chacun le même degré de lumière. En cela, il est plus «moderne» en effet que nombre de philosophes postérieurs.

(10) Voir les propos de Lesbros de la Versanne rapportés dans la notice du Cabinet du Philosophe: «Je lis les Pensées de Pascal, répondit-il. M. de Marivaux pensait que c'était le meilleur livre de morale qui eût jamais été écrit.», dans Journaux et Euvres diverses, op. cit., p. 329.

(11) Cette expression est évidemment un clin d'œil en direction de l'ouvrage de P. BOuRDieu portant ce titre (Paris, Le Seuil, 1966), et dans lequel il pose d'ailleurs les mêmes problèmes épistémologiques qu'aborde Marivaux, selon nous, quant à l'implication de l'observateur dans la constitution du phénomène social observé. Voir plus loin la question de la misanthropie. 
Il y a là, «tout mêlé entre les mots» comme dirait Proust, tout le mécanisme d'auto persuasion des spectateurs, contenu dans les figures de duplication et d'ellipse, faisant passer de la probabilité à la ferme conviction: cela doit être beau, donc j'admire! Cette sorte de cogito de la dupe apparente souligne évidemment les prodiges de l'imagination. Marivaux expose les pensées du peuple, les «développe» (le terme sera plus loin), va des «principes» à la «forme», comme Pascal aussi s'attachait à manifester la logique inaperçue et subreptice des comportements. L'entrée en posture d'admiration est mécanique: l'interrogation rhétorique «vois-tu ce monde?» fait fonction de stimulus, l'appel à la curiosité soulignant le côté «pavlovien» des badauds. De quoi il résulte un mixte de voyeurisme, de comportement de moutons de Panurge, de jouissance à se saisir de ce qui paraît donné «gratis», de connivence fantasmée avec l'Infante en l'honneur de qui est donnée la fête, l'ensemble aboutissant à cette tautologie: le peuple admire... par admiration. Comme au théâtre, le peuple jouit non du fait de ce qu'il y aurait réellement d'admirable mais à cause de la machinerie à produire de l'imaginaire sur lequel repose cet événement, qui n'en est un que pour celui qui est en quête d'idées, le Spectateur. Pourtant, il y a plus de tendresse que d'ironie dans l'assimilation entre les deux machines spectaculaires, celles des gens de théâtre et celle des politiques, et le personnage de Marivaux comprend les spectateurs plus qu'il ne les condamne: «Il est certain que ces arcs de triomphe étaient curieux, et que c'était une décoration qui avait beaucoup de dignité [...]», concède-t-il. Avant d'effectuer un autre saut, de procéder à un nouvel éloignement du point de vue:

[...] mais, en développant l'esprit de cette populace, je voyais de pauvres enseignes de cabaret à qui, peut-être, il ne manque pour être converties en chefs-d'œuvre, que d'être exposées dans une aventure de conséquence.

Tableaux de Raphaël! disais-je encore en moi-même, si vous étiez à la place de ces mêmes enseignes, $\mathrm{j}$ 'aurais grande peur que vos curieux ne vous prissent pour ce que vous paraîtriez. Je veux mourir si en vous voyant ils s'avisaient de vous deviner là.

Pourquoi cet apparent coq-à-l'âne? Pourquoi évoquer des enseignes de cabaret, puis la peinture de Raphaël? Il y a une équation implicite: une enseigne est-elle à un Raphaël ce qu'une personne anonyme est à un Grand? Et sur quoi repose la distinction? Marivaux est encore dans le double registre. Il est censé décrire le peuple et la rue, et il s'y trouve probablement des enseignes de boutiques. C'est la dimension référentielle, à laquelle il tient, à juste titre pour la valeur de sa démonstration. Elle requiert la simple posture de «reportage», et des enseignes qui soient aussi des tableaux de maître, l'époque en est coutumière. Watteau, en 1720, venait de peindre celle de son ami marchand d'art Gersaint. Chardin lui aussi donnera notamment une enseigne de chirurgien, une autre pour un commerce de billards, profession exercée par son père. Marivaux s'amuse d'une mode et, en moderne, s'intéresse à l'éphémère, au passager (d'ailleurs ce genre pictural sera un pan oublié de l'histoire de l'art...). Mais l'autre registre est de l'ordre de l'intertexte pascalien sous-jacent, qu'il faut ici rapidement décliner. ${ }^{12}$ "L'empire fondé sur l'opinion et l'imagination règne quelque temps, et cet empire est doux et volontaire» (fragment 546): cette idée selon laquelle la société des hommes repose sur un imaginaire impérieux plus suave que n'est la force, mais à l'usage fort ambigu, est capitale dans toute cette feuille. Il faudrait aussi mentionner le célèbre fragment 78 , sur l'imagination qui «donne le respect et la vé-

(12) Les références qui suivent aux Pensées de B. PASCAL seront données selon le classement de l'édi- tion P. SELliER, Paris, Bordas, 1991 («Classiques Garnier»). 
nération aux personnes, aux ouvrages, aux lois, aux grands», et qui «dispose de tout. Elle fait la beauté, la justice et le bonheur qui est le tout du monde». Dans ce même fragment se trouve l'histoire du prédicateur mal rasé par son barbier, laquelle engendrera dans notre feuille du «Spectateur», deux paragraphes plus loin, l'apparente digression opposant un premier homme «plein d'esprit et de talent», mais invisible tel un fantôme, à un second homme, mis en valeur, lui, grâce au tableau d'un «barbouilleur»:

C'est un personnage inutile, confondu dans la foule, que nous méprisons; il n'a ni biens, ni rang, ni crédit; voilà le fantôme qui nous frappe à la place de l'homme que nous n'apercevons pas; voilà le masque qui nous cache son visage; enfin voilà le tableau, tout beau qu'il est, enseigne de cabaret pour toujours. Tel au contraire est un tableau de barbouilleur; et je le vois entouré de curieux qui lui trouvent un vrai mérite qu'il n'a point. Est-il pesant? parle-t-il peu? Ils me disent que c'est un homme froid mais plein de jugement et de réflexion. Parle-t-il beaucoup? qu'il est agréable et vif!

Mieux vaut donc un imbécile rendu séduisant par un peintre médiocre qu'un sage que rien ne signale à l'attention de la foule. Inversement: «Tout l'éclat des grandeurs n'a point de lustre pour les gens qui sont dans les recherches de l'esprit» (fragment 339). Telle est la justification du fait que Marivaux se dispense obstinément de l'ekphrasis que son sujet aurait dû impliquer: s'il ne donne au lecteur rien à voir, c'est que les idées à glaner ne sont pas de l'ordre du visible. Mais alors, pourquoi ne pas se contenter de la posture du moraliste classique? Pourquoi se faire spectateur, aller voir le peuple, dans la rue, si c'est pour en repartir tout dépité quant aux hommes?

Il y a deux réponses à ces questions. Souvenons-nous tout d'abord que chez Pascal cette critique de l'imagination est largement reprise de Montaigne, et donc ne représente que le niveau de lecture des sceptiques (fragment 124: «Le peuple honore les personnes de grande naissance. Les demi habiles les méprisent, etc.»). Par «demi habiles», entendons: le sceptique. Ce serait une erreur que de croire que Marivaux déprécie les Grands ou le spectacle donné au peuple (même désigné comme «populace») alors qu'il n'est question que d'illustrer les prodiges dont l'imagination est capable et d'en interroger le sens profond. Rappelons que (fragment 134) le peuple a les opinions très saines d'avoir choisi le tumulte, le jeu, le divertissement, mais pour une raison qu'il ne pénètre pas. À un degré de discernement supérieur, l'on peut dire que si les êtres humains sont fascinés par l'imagination qui les divertit, du moins le sage peut-il jouir de l'observation des signes comportementaux que les hommes produisent et grâce auxquels ils règlent sans même en avoir conscience leur vie sociale. D'où la nécessité de se doter d'une sémiotique, d'un outil, même élémentaire, permettant de distinguer les signes, et de penser leurs fonctions. Cette sémiotique, c'est l'image de l'enseigne qui l'incarne et l'exemplifie dans le discours du Spectateur. Elle vient encore de Pascal (fragment 486): «On ne passe point dans le monde pour se connaître en vers si l'on n'a mis l'enseigne de poète, de mathématicien, etc. Mais les gens universels ne veulent point d'enseigne, et ne mettent guère de différence entre le métier de poète et celui de brodeur.» L'enseigne des rues est bien sémaphore, porte signes, ce par quoi l'on se signale, et se signaler c'est exister à la vue et l'attention des hommes, lesquels ne connaissent d'ailleurs du juste, du vrai et de tout le reste que des signes. Dont s'empressent de se parer le Juge, le Médecin, le Roi même, qui «sans divertissement est un homme plein de misère» (fragment 169). Le Roi auquel les courtisans apprennent à jouer et danser afin qu'il ne ressente pas le vide qui l'entoure et le constitue...

Et de la même manière que Pascal légitimait ironiquement le rôle de l'homme de cour, toute la fin de cette partie va consister pour le Spectateur de Marivaux à justifier l'attitude des flatteurs eux-mêmes, puisqu'ils 
[...] sont en cela de vraies dupes, de vrais innocents, dont l'esprit est, pour ainsi dire, aux gages de l'intérêt. C'est ce misérable intérêt qui a joué ce tour de souplesse à leur jugement, et qui leur a fait accroire qu'un grand équipage, un grand nombre de valets, une bonne table sont de l'esprit, de la pénétration, et de vivacité et de bons mots $^{13}$.

Tant dans le spectacle de la vanité qu'en constatant la vanité du spectacle, il y a malgré tout de quoi admirer et se réjouir, fût-ce seulement d'observer ce mécanisme dénommé par Marivaux «tour de souplesse»!... Pour être signalé aux yeux des hommes, il faut donc un cadre. N'est visible que ce qui est sélectivement désigné aux yeux, à tel point que souvent la valeur de l'objet vu est déduite de celle du cadre qui en permet la vision. «C'est l'enseigne qui fait la chalandise», écrivait aussi La Fontaine. C'est pourquoi, en poussant le raisonnement à la limite (ou en direction de l'infini, pour reprendre un terme pascalien), une enseigne de cabaret vaut absolument une peinture de Raphaël, dans l'ordre esthétique, et pourquoi, dans l'ordre éthique «nous sommes tous des tableaux les uns pour les autres». Tel est ce par quoi conclut provisoirement le Spectateur.

Chez Pascal également, les niveaux éthique, politique et esthétique étaient étroitement combinés (fragment 78: «la vénération aux personnes, aux ouvrages... l'imagination fait la beauté, la justice»). Il en va de même dans le texte de Marivaux, ce qui lève la difficulté que nous avions à comprendre comment il peut associer - de façon si incongrue pour nous - l'enseigne peinte de la rue avec le tableau de Raphaël. D'où la nécessité de passer, dans la scène suivante, du grand peintre au tableau du Grand par excellence, le Roi. Ce sera la troisième scène. Et ce sera la deuxième réponse à la question qui se posait plus haut. Car Marivaux peut-il se contenter de proposer un point de vue relativiste, et finalement amer, sur le discernement dont est capable le peuple spectateur, donc sur la valeur intrinsèque du spectacle, qu'il soit politique ou théâtral? N'y a-t-il pas au contraire, dans la composition scripturaire qu'il a suivie, et que l'on pourrait dénommer un recul progressif du point de vision, l'indice qu'il convient de rechercher aux phénomènes décrits une motivation ultime, grâce à une forme de «pensée de derrière»? S'il suit effectivement une problématique toute pascalienne, dans l'étagement qu'il pose entre les niveaux d'interprétation, Marivaux ne sera pas pascalien jusqu'au sens du tragique...

\section{Scène 3: L'apparition du Roi, éludée sitôt que mentionnée}

Jusqu'à présent, le Spectateur donnait essentiellement ses propres méditations, et rien de l'objet spectaculaire. Avec «C'était là à peu près les idées qui me venaient successivement dans la tête, quand le roi a passé», l'on s'attend à ce qu'enfin vienne le tableau vivant, l'apparition attendue. Marivaux poursuit:

Le peuple, à son ordinaire, a crié Vive le roi. J'ai trouvé ses acclamations attendrissantes. C'était plus qu'un roi, plus qu'un maître qui paraissait. Ce peuple, dans ses transports, semblait revêtir ce jeune prince de titres moins superbes, mais plus aimables, plus touchants, et peut-être plus augustes: c'était le bienfaiteur, l'ami de chaque homme de la nation; c'était le protecteur, l'espérance, l'amour et les délices du peuple que l'on voyait passer.

Il n'est plus question de «populace» mais bien du «peuple», compris comme constituant essentiel de la «nation». Marivaux place en exergue des termes dont il

(13) Nous soulignons. Notons là encore la proximité de ces expressions avec des répliques d'Arle-

quin dans La double inconstance. 
n'ignore rien de la valeur tant dans la tradition théâtrale qu'en philosophie politique: «superbe», «auguste» renvoient à la représentation cornélienne de la fonction royale, et par-delà aux réflexions notamment d'un Jean Bodin sur la souveraineté dans ses rapports avec la constitution d'une nation. Mais il tempère ce vocabulaire lourdement chargé de l'isotopie de la puissance par un autre registre, celui de l'affection amoureuse: «aimables», «touchants», «ami», «amour», «délices». Manière de décentrer la définition de la souveraineté en même temps qu'il vient de déporter l'attention du lecteur: car, du roi, toujours rien, ou plutôt il apparaît dissous dans ces métaphores allant croissant en abstraction: «c'était le protecteur, l'espérance, l'amour et les délices du peuple que l'on voyait passer.» Au tableau du jeune Roi, Marivaux substitue donc le défilé allégorique des qualités que l'on attend désormais d'un roi. C'est encore le peuple qui intéresse le Spectateur, lequel s'attendrit à la vue des vivats quand on se serait attendu à ce qu'il fît preuve de dédain: loin d'être passif en effet, ce peuple «semblait revêtir» la figure du roi des qualités dont il attend que ce dernier fasse preuve, et en ce sens ses acclamations révèlent non une duperie mais bien la conscience qu'il signe une forme de contrat. Alors, s'interposant brutalement entre peuple et prince, le Spectateur enchaîne sur un morceau d'éloquence commençant par cette apostrophe:

Rois, princes de la terre! ce n'est ni la garde qui vous environne, ni cette foule d'hommes soumis qui composent votre cour, ni vos richesses, ni votre vaste puissance, qui feraient mon envie. Ceux qui, parmi vous, ne sont sensibles qu'à ces avantages sont simplement des hommes riches, redoutables, puissants, et ne sont pas rois.

Bien entendu, le Spectateur ne prend la parole que fictivement, et l'on croit percevoir un net décalage avec la norme supposée de la relation d'un événement, avec le modèle «journalistique» que Marivaux semblait suivre. Pour autant, apostropher le prince, n'est-ce pas relayer le sentiment populaire, servir d'interface entre le monarque et cette entité que les Lumières nommeront bientôt le «vrai souverain»? C'est là l'un des aspects du fait journalistique, compris et assumé par Marivaux. Mais plus profondément il se situe aussi dans une logique déjà ancienne de la relation directe entre prince et peuple. Comme c'était le cas plus haut avec la référence implicite à La Fontaine, le long paragraphe qui suit est inspiré d'une topique littéraire, celle de l'adresse au Roi, telle qu'elle pouvait se pratiquer dans les cérémonies des entrées royales. Guenée et Lehoux en soulignent parfaitement les dimensions spécifiques. «Une entrée, comparée à un sacre ou un enterrement, est une bien modeste chose. Mais un roi, au cours de son règne, a fait des dizaines d'entrées, qui ont sans doute au total, mieux que son sacre, assuré son trône» ${ }^{14}$. L'enjeu est donc bien de renouer le pacte de souveraineté. Il faut donc y impliquer le peuple, comme spectateur et acteur à la fois:

Dans une entrée [...], on tient certes compte de la volonté royale et de la tradition, mais ce sont les bourgeois de la bonne ville qui l'organisent; ce sont eux, leurs femmes et leurs enfants qui en sont les acteurs. Une entrée est donc, mieux qu'un sacre, l'occasion d'un dialogue entre un roi plus proche et des sujets moins passifs ${ }^{15}$.

Certes, ce n'est pas le jeune Louis XV qui entre dans sa bonne ville de Paris, c'est l'Infante qui l'y retrouve, mais, par sa personne, ce sont bien la paix retrouvée, les

(14) B. GuenÉE et F. LEHOux, Les entrées royales françaises de 1328 à 1515, Paris, éditions du CNRS,
1968 , p. 8.

(15) Ibid. 
puissances réconciliées et la fécondité de la lignée projetée, dont il s'agit de pénétrer les cœurs et les esprits. Le Spectateur, donnant cette adresse au roi, obéit en réalité au jeu d'un rituel alliant «spectacle pittoresque et solennités grandioses» ${ }^{16}$, au cours duquel voir le roi, être proche de lui, lui parler même, importent au moins autant que, pour le peuple, de se percevoir comme constituant une entité unanimement aimante et aimée. Et l'on est bien dans la même logique que celle des cérémonies d'entrées, au cours desquelles le roi était amené à renouveler solennellement les privilèges de telle ou telle corporation, ou bien à proférer tel serment de fidélité et continuité, donc à entendre les exigences de son peuple. Alors, le Spectateur poursuit:

Rois! Qu'est-ce donc que votre condition a de flatteur? Quel est celui qui règne? Quel est le prince qui jouit des vrais biens attachés au trône? C'est celui qui sait faire un généreux usage de la crainte et du respect que la majesté de son rang inspire; cette crainte et ce respect sont les moindres de ses droits, ou plutôt ils ne font que lui préparer ses véritables droits. Craint, il n'est encore que le maitre; aimé, le voilà roi.

Si l'admonestation initiale, rappelant que le vrai roi n'est pas sur terre et que les princes ne règnent que par sa délégation, n'est pas originale, en revanche à la fin de ce passage Marivaux se démarque nettement de Pascal, pose sa propre philosophie politique. Pascal affirmait que les magistrats et les médecins ont besoin de se déguiser, avec un «appareil auguste», et non point les rois (car ils ont les hallebardes, la force). Dans le roi, ce qui inspire le respect, c'est l'expression de la terreur (voir les fragments 59, 60: «La puissance des rois est fondée sur la raison et sur la folie des peuples, et bien plus sur la folie»). Marivaux en revanche gomme toute mention de la puissance (pourtant elle aussi spectaculaire, et d'ailleurs présente dans la gravure anonyme), à moins que justement il ne conteste sur ce point son maître à penser. Marivaux élimine les composantes tragiques de terreur et de folie du peuple, en centrant sa réflexion sur l'amour exclusivement, et ce dans un vocabulaire rappelant celui de ses propres comédies, en donnant aux princes une leçon de politique reposant sur une sorte de «bon usage» de l'amour populaire:

Et comment l'aime-t-on? Comptez tous les sentiments de vénération, d'estime, d'admiration; tous les mouvements de tendresse, de dévouement, de confiance, dont l'homme est capable: voilà de quoi se compose l'amour qu'on a pour un maître dans qui l'on est charmé de trouver un roi; enfin, voilà les trésors du rang suprême. Un accueil obligeant, un sentiment de bonté, un sourire, un geste, une parole; princes! ce sont là pour vous les clés de ces trésors.

Dans son troisième Discours sur la condition des grands, Pascal distinguait le roi par charité du roi par concupiscence, Dieu seul incarnant le premier, tous les autres rois terrestres ne faisant qu'administrer la concupiscence de leurs sujets ${ }^{17}$. Le dialogue sous-jacent que, depuis le début de cette feuille, Marivaux a entretenu avec Pascal, l'a conduit à inverser la perspective janséniste.

Rappelons-en les étapes. Faut-il assister à ce genre de cérémonie? Le Savetier d'abord nous en dissuade. Il suffit de parcourir les rues pour voir le peuple, ébahi devant les pompes comme face à un tableau illusionniste, sous l'emprise de l'imagi-

(16) Ibid. La thématique sentimentale de l'amour n'est pas absente des cérémonies d'entrées. Ainsi en 1498, pour celle de Louis XII à Paris, une jeune fille nommée Bonne Volonté menant un cerf volant s'adressa-t-elle au roi en ces termes: «A vostre venue excellente/ Le cerf volant je vous presente/ Af- fin que la ville desserve/ Votre amour a vous se rend serve/ Et de tous biens obediente». Voir p. 132.

(17) Pascal, Euvres complètes, édition Jacques Chevalier, Paris, Gallimard, 1954 («Bibliothèque de la Pléiade»), p. 620. 
nation. Or, «quelle vanité que la peinture, qui attire l'admiration par la ressemblance des choses dont on n'admire pas les originaux» (fragment 74). Pour un pascalien de stricte obédience, une enseigne vaudrait effectivement un Raphaël, prouvant les pouvoirs littéralement «fous» de l'imaginaire, lequel en retour nous tend l'image de notre vanité. Déjà, Marivaux est moins relativiste que Pascal: en admettant qu'un tableau ne vaudrait que par le cadre qui le signale à notre estime, il pousse l'exemple à la limite (suivant Pascal encore en cette démarche même) en prenant ceux de Raphaël, qui sont finalement bel et bien distincts, pour le Spectateur, des «pauvres enseignes de cabaret», pour lesquelles il ne dissimule pas non plus une certaine tendresse. Car les déprécier, ce serait aussi mépriser ces hommes sans distinction, ceux qui sont comme des tableaux envers lesquels notre jugement varie du pour au contre selon le type d'espace social qu'ils occupent, par lequel ils se signalent. Quand Pascal voyait dans la sémiotique sociale la manifestation du vide intrinsèque de la vie en commun, Marivaux au contraire va s'en emparer pour la valoriser. Savoir lire et former incessamment des signes, quelle plus belle manifestation de l'intelligence et de l'inventivité sociale dont font preuve les hommes?

Renversant les conclusions de la dialectique du divertissement, qui fait du peuple une dupe éternelle et de son souverain un être «plein de misère», il s'adresse alors au roi. Certes, le roi, loin de se trouver au-dessus du lot des hommes, pourrait n'incarner qu'un être factice, ne donner qu'une image ironique de la vacuité commune; le rituel de la présentation solennelle pourrait fonctionner à vide. L'emprunt de la topique de l'entrée royale permet au Spectateur de lancer son adresse à valeur éminemment phatique, de convoquer en somme le «corps du roi», présent (occasion rare) au milieu de son peuple. Et tandis que Pascal avait finalement dissout le lien politique, au contraire Marivaux en restaure fermement la nécessité, convoquant, sur la même scène, le peuple acteur et le roi spectaculaire. Entre eux, il faut inventer un rapport d'échange, une monnaie symbolique pour sortir du cercle de la fascination stérile. Cette monnaie, ce sera l'amour. Car être aimé, pour un Prince, cela crée des obligations. Il s'agit pour le roi d'apprendre à ne pas méconnaître les devoirs que lui impose l'expression des sentiments hyperboliques que le peuple projette en lui. De le payer en retour dans la seule monnaie qui vaille entre les hommes, l'amour. Le travail du prince, le seul sans doute, mais le plus difficile qui soit, est de savoir mériter d'être aimé, et via ce sentiment (qu'il doit savoir vivifier et renvoyer en miroir) de constituer son peuple en nation unie. L'affection que lui exprime le peuple - et rappelons ici qu'à cette époque, et pour quelque temps encore, Louis XV bénéficie du surnom de «bien-aimé» - ne lui est pas due, elle n'est que l'expression préliminaire à la prise de conscience de ce que sont «ses véritables droits.»

Marivaux est ici autant théoricien du théâtre que philosophe politique; et même, il est l'un par l'autre. C'est d'un seul élan qu'il fonde la nécessité politique de la scène théâtrale et la valeur théâtrale de la scène politique. D'abord, «cette crainte et ce respect» qu'il évoque pour les récuser sont les catégories qu'emploient les théoriciens du genre tragique. Il est sûr que Marivaux ne souhaite pas ce type de pouvoir-là ${ }^{18}$. Quant au mode d'emploi de l'amour, il évoque irrésistiblement celui qu'il mettra en œuvre aussi dans La double inconstance. Lorsqu'Arlequin accepte de donner Silvia

(18) Dans la tradition de la philosophie politique en France, et en particulier chez un Jean Bodin, la réflexion sur le fondement et l'exercice de la souveraineté se concentre sur les notions de force, de crainte et de respect. Le respect est évidemment la distance («incommodez-vous», le définit Pascal), tout le contraire de la notion d'amour qu'avance ici
Marivaux. Cette dernière n'est pourtant pas absente des réflexions des philosophes humanistes, notamment chez Erasme qui écrit: «Le tyran cherche à inspirer la crainte et le roi l'affection» (cité par $\mathrm{P}$. MESNARD, L'essor de la philosophie politique au XVI siècle, Paris, Vrin, 1977, p. 121). 
au Prince en reconnaissance de sa bonté («vous avez tant de charité pour moi, n'en aurai-je pas pour vous?»), le Prince, révoquant l'hypothèse d'un Arlequin qui serait devenu calculateur et courtisan, lui répond: «Nous nous brouillerons ensemble si tu ne me réponds toujours ce que tu penses. Il ne me reste qu'une chose à te dire, Arlequin: souviens-toi que je t'aime.» (III, 5). Virulente admonestation dans la feuille du «Spectateur»; confrontation agressive dans la pièce de théâtre: l'amour entre un sujet et son prince n'est pas donné a priori, au contraire, puisque dans cette pièce tout commence par la violence politique qui a consisté en l'enlèvement de Silvia. Mais c'est bien au prince, y compris en se faisant violence à lui-même, de savoir gagner le cœur de son peuple et de ne pas mésuser de l'affection que ce dernier lui donne! Ajoutons ceci d'essentiel: le «je t'aime» proféré dans La double inconstance par le Prince en direction d'Arlequin n'équivaut pas à exprimer de la reconnaissance, ni de la gratitude. C'est bien d'amour qu'il s'agit, du sentiment le plus fort qui se puisse concevoir entre deux être humains, et dont l'effet premier est de rendre l'un absolument symétrique de l'autre. Mais pas identique. Arlequin n'est pas devenu par la magie du verbe l'équivalent ni l'égal de son maître. Le Prince doit avoir assez de discernement pour connaitre que rien ne le distingue par nature d'un Arlequin. Et cette convention qui fait de l'un un prince, de l'autre un valet, une fois connue comme telle, permet d'une part à Arlequin de faire allégeance au Prince sans s'humilier, d'autre part au Prince de jouer sa fonction de pôle fédérateur de la communauté sans tomber dans le piège qui guette tous les Grands: s'imaginer stupidement être fait d'une autre essence que le premier quidam venu (et Arlequin est bien, socialement parlant, un quidam!). Autrement dit, il y a bien chez Marivaux une conception, partiellement héritée de Pascal, faisant de tout statut social une pure convention, et qui pour être arbitraire n'en est pas moins vitalement nécessaire: par nature le prince n'est évidemment pas différent du dernier de ses sujets, mais l'un et l'autre doivent faire comme si le prince l'était pourtant. Le peuple, surtout, a tout à gagner à cette fiction: s'unir afin d'être plus fort, limiter le nombre des tyrans, éviter la guerre civile, etc. Qu'est-ce qui fait l'unité du peuple? Sa capacité à se projeter en un tout doté d'un même avenir. La personne du prince ne fait que matérialiser cet ensemble que la cérémonie clame et magnifie pompeusement.

Se projeter: nous sommes encore dans une question de scène et d'optique. Aussi bien, la «populace» a-t-elle raison de s'émerveiller des arcs, des tableaux qui lui sont proposés, non parce qu'en soi ils sont beaux ni parce que ceux qu'ils honorent seraient différents de tout un chacun, mais en ce que ce décorum invite le peuple à donner son amour au roi et le roi à se donner au peuple. À faire le don au moins de son image, de sa «vue», qui n'a d'intérêt que de permettre au peuple de se voir comme «tout» incarné en un seul, réuni... Finalement, cette cérémonie a valeur de test: si le jeune roi comprend bien que les affects du peuple (son admiration, sa joie naïve) ne sont nullement méprisables, c'est tout simplement qu'il saura l'aimer, qu'il sera digne de ce qui fictivement le distingue ${ }^{19}$. Par conséquent - et c'est l'aspect propitiatoire de la cérémonie - cela veut dire aussi qu'il accomplira les espérances que

(19) Dans L'éducation d'un Prince, qu'il publie dans le Mercure en décembre 1754 (trente ans après son Spectateur français), Marivaux revient sur ces problèmes, qui manifestement lui tiennent à cœur. Au jeune prince Théodose, qui imagine que sa noblesse est biologique («Quoi, le sang dont je sors...»), son précepteur Théophile, le coupant, rétorque: «... est consacré à nos respects et devenu le plus noble sang du monde; les hommes se sont fait et ont dû se faire une loi inviolable de le respecter; voilà ce qui vous met au-dessus de nous. Mais dans la nature, votre sang, le mien, celui de tous les hommes, c'est la même chose; nous le tirons tous d'une source commune.» Une fois la leçon comprise, Théodose affirme à son tour: «La nature ne fait que des hommes et point des princes; je conçois maintenant d'où mes droits tirent leur origine.» Voir Journaux et Euvres diverses, cit., p. 522 et p. 526. 
le peuple projette en lui, qu'il saura être, non un maittre, un roi, sachant donner des preuves d'amour: ces mêmes gages dont les personnages des comédies de Marivaux ne cessent de vouloir s'assurer...

Est-il donc convenable de faire admirer le Roi grâce à ce genre de cérémonie ? N'est-ce pas mépriser le peuple et dévaloriser le prince que de les impliquer dans un tel divertissement? Non seulement c'est légitime, mais il est nécessaire que le peuple soit invité à valider activement la fonction du jeune roi. Par une sorte de ruse de l'imagination et de la raison réunies, l'admiration a priori stupide du peuple pour ce spectacle aura accompli sa vocation: permettre l'expression de l'amour, que le prince exprime publiquement le sien comme cause et reflet de celui du peuple. Réciprocité, symétrie, jeux de miroirs: ici, les effets d'optique sont aussi des constructions politiques. L'affection populaire, si «fleur bleue» quelle paraisse (l'espérance, l'amour et les délices) presse en retour le prince d'exprimer une aptitude à aimer symétrique et proportionnelle: «soyez doux, affable, généreux.» Selon l'étymologie (genus), la générosité du prince est le signe de celui qui est bien né; ce qui valide sa distinction nobiliaire, fictive en référence à la nature, essentielle à la communauté politique en ce qu'elle pose une inégalité fonctionnelle dont nul ne doit être la dupe, et surtout pas le prince s'il connaît ses devoirs. Encore faut-il les lui rappeler: ce à quoi s'emploie si vivement le Spectateur, sortant de sa supposée neutralité, devenant intercesseur pour exprimer en première personne un discours qui n'est autre que la projection de la conscience collective, voire d'une sorte de «volonté générale».

La fin de cette feuille va alors tenter de penser l'articulation entre cette conscience et l'opinion publique.

\section{Scène 4: Ce qu'ils voient? C'est ce qui les regarde...}

De même que, sortant du théâtre, les spectateurs échangent impressions, sentiments et critiques, ainsi, le roi étant passé (mais sans qu'il ait été décrit au lecteur du «Spectateur français»!), le personnage de Marivaux va pouvoir engranger de nouvelles observations. La tactique narrative, faite de reculs successifs, d'une cascade de points de vue presque aléatoires, parvient alors à une phase conclusive.

Quelqu'un que j'ai entendu parler alors, d'un ton de voix extrêmement haut, a mis fin à mes réflexions. Là-dessus, je me suis retourné et j'ai vu plusieurs hommes qui en entouraient un autre qui leur parlait avec beaucoup d'action. J'ai soupçonné qu'il y aurait là quelque chose pour moi. Je me suis donc rapproché [...].

Si anachronique que le «journalisme» de Marivaux puisse paraître, il y a dans la composition de cette feuille une forme de conscience de ce que doit être l'unité d'un véritable «reportage». Car il ne se contente pas, en simple chroniqueur, de noter les faits. Il s'intéresse également à la perception qu'en a le public: loin de terminer son récit une fois l'acmé événementielle passée, il instaure une continuité entre le spectacle et sa réception, procède par phases synchrones avec la durée de l'événement d'ensemble. Après le moment culminant de l'exhortation adressée au roi, le narrateur recadre en direction du public, capte une nouvelle scène. On avait vu le peuple se préparer à admirer le spectacle, on va recueillir ses impressions, et poser incidemment la question clé: dans quelle mesure et comment le peuple est-il apte à élaborer un jugement politique viable?

$\mathrm{Au}$ discours indirect, viennent deux saynètes ayant en commun le pittoresque. Un premier homme est saisi, qui «parlait de la dernière paix avec l'Allemagne et l'Angleterre», qui glose sur l'habileté des ministres, s'enflamme. Le Spectateur s'intéresse 
non tant à la nature des propos de cet homme qu'aux effets que produit sur lui-même sa propre éloquence:

De la façon dont cela se passait dans son esprit, je voyais que c'était lui qui se réconciliait avec les puissances, ou plutôt il était tour à tour l'Allemagne, l'Angleterre, la Hollande et la France. Il avait fait la guerre, il faisait la paix. L'admiration judicieuse qu'il avait pour les ministres lui en glissait une de la même valeur pour lui-même.

Nous sommes toujours sur le même double registre que depuis le début du texte. Nous avons d'abord un témoignage relatif à la perception de l'actualité par ses contemporains, comme il en existe d'ailleurs depuis la fin du XVI siècle:

Sous le titre des Passionnés de guerre, une gravure bolonaise de 1690 met en scène le nouvelliste, assis dans la rue, de grosses lunettes sur le nez, en train de lire à une quinzaine de personnages agglutinés autour de lui et parmi lesquels le graveur a pris soin de placer des représentants de toutes les catégories de la société urbaine. Les physionomies sont attentives, les exclamations fusent, une rixe en arrière-plan oppose deux des auditeurs aux opinions visiblement divergentes ${ }^{20}$.

Ce qui atteste que l'expression publique (dans la rue) des opinions relatives à l'actualité n'est pas nouvelle, surtout dans les villes universitaires où s'est constituée ce qu'Habermas dénomme la «bourgeoisie des talents» (Bildungsbürgentum). L'on pourrait même dire que le pittoresque de la scène décrite par Marivaux tient à ce que la dispute entre gens du peuple est déjà un cliché, et littéraire, et iconographique. Forme de second degré, donc, une fois encore. Mais double registre aussi, car c'est toujours un mode de raisonnement pascalien qui intéresse Marivaux. Que son personnage ait raison ou tort, qu'il persifle, cancane ou déblatère lui importe peu: le Spectateur se concentre sur les effets de la formation de l'opinion sur le comportement de celui qui l'élabore. L'on pénètre en l'esprit du personnage:

Bientôt les ministres et lui ne faisaient plus qu'un, sans qu'il s'en doutât. Je sentais que dans son intérieur il parcourait superbement un vaste champ de vues politiques.

C'est le phénomène de l'identification qui est mis en évidence: l'homme qui a attiré l'attention du Spectateur est une espèce d'acteur au naturel, il a créé un attroupement autour de sa personne, s'est échauffé jusqu'à l'enthousiasme, est habité par les rôles qu'il endosse, par les idées qu'il remue, les spéculations qu'il conçoit. L'on est au théâtre: aussi bien, Marivaux se garde-t-il de se moquer d'un homme si manifestement manipulé par les fruits de sa propre imagination. Une fois encore, la démarcation d'avec Pascal est claire. Mais, plus intéressante est la manière dont Marivaux assume pleinement le parallélisme entre la rue fonctionnant comme scène politique, d'un côté, et de l'autre les idées politiques comme effets d'une mise en scène de l'apparition du roi, ainsi que des corps et des paroles des spectateurs. Ces idées portent un nom dans ce texte: «vaste champ de vues». Expression qui associe les deux significations du mot «vue», la conception idéelle et le dispositif visuel. Ce recoupement sémantique entre l'idée et le tableau, entre les spéculations et ce qu'en langue italienne on dénomme «veduta», où sont représentées des villes conçues comme des scènes de théâtre, cette association étroite, systématique et concertée, entre les domaines optique et scénique, spéculaire et spéculatif ne saurait être fortuite. 
Une «vue» suppose un point de vue, c'est-à-dire un sujet pensant, comme le rappelle clairement Hubert Damisch:

La perspective ne joue pas sur le seul registre de l'imaginaire [...]; elle a ceci de commun avec la langue, qu'en elle et par elle, s'institue, se constitue sous l'espèce d'un point, une instance analogue à celle, dans la langue, du "sujet", de la "personne", toujours posée en relation avec un "ici" ou un "là", avec toutes les possibilités de passage d'une position à l'autre qui en résultent ${ }^{21}$.

Dès lors, les décentrements successifs de la vision qu'on a notés depuis le début de cette Feuille V prennent tout leur sens: Marivaux est pleinement conscient de ce que rapporter un fait, c'est le construire, et il élabore son reportage comme une suite de scènes, lesquelles se superposent tout naturellement à la scénographie de l'entrée royale. Mais au-delà, c'est la question de la mise en perspective qui se pose, comme construction artificielle disposant l'équivalence entre un point de vue et un cogito ${ }^{22}$, positionnant un sujet de la vision face à un plan qui est lui-même articulé et dédoublé de sorte qu'au-delà de ce plan se pose virtuellement un point de fuite qui est l'équivalent du point de vision. Autrement dit, chaque modification successive de la scène décrite induit un changement de construction du point de vue ainsi que du point de fuite, étant donnée la solidarité entre l'un et l'autre. Or il semble que ce dispositif, qui est bien connu dans l'histoire de l'art, est ici d'un intérêt particulier, dans la mesure où ce texte pose la question de la constitution conjointe d'un point de vue sur un événement et de l'apparition parmi son public d'une vision critique à son égard, collective et individuelle. Les deux acceptions de la notion de «point de vue» sont par conséquent étroitement corrélées: le dispositif optique et la formation de l'opinion. Et d'une certaine façon, si le Spectateur ne décrit rien de ce qu'on aurait dû voir, c'est, depuis le début, parce que sont imbriqués le problème de son inscription face à l'événement et celui de la fonction du spectaculaire, de ses conséquences en termes de passions et de réflexions. Ce que le Spectateur voit est déterminé par la nature de la construction optique qu'il dispose, potentiellement tous azimuts; mais aussi cette dernière est-elle également fonction du type de scène qui s'impose aléatoirement à lui. Un réglage réciproque et souple constitue donc le rapport à l'événement, le jugement s'élabore en tant que lié à ce réglage. Et ce qui ressort de cette structure originale, c'est une question analogue à celle formulée par Hubert Damisch: «Qu'est-ce que voir, si ce que je vois me regarde? $\gg^{23}$.

En d'autres termes, ce que Marivaux met ici à jour, c'est la structure en miroir de l'événement politique, dès lors qu'il cesse d'être conçu comme monodirectionnel, que les spectateurs ne sont pas les destinataires passifs et éberlués mais bien les concepteurs d'une opinion publique assumée et finalement libre (ayant y compris la liberté de ne pas assister au spectacle: c'était l'option du Savetier). Ce qu'ils voient c'est ce qui les regarde (les concerne), et le plus important a son lieu non pas sur la scène mais bien dans l'esprit des spectateurs, le spectacle passé.

Qu'il y ait à la base de cette réflexion une fois encore le modèle théâtral, c'est probable. Mais Marivaux l'élargit considérablement. L'opinion qui germe dans l'es-

(21) H. Damisch, L'origine de la perspective, Paris, Flammarion, 1987 («Idées et recherches»), p. 62 .

(22) «Les recherches sur la perspective témoignent en effet d'un intérêt pour la vision dont on ne peut pas ne pas voir (c'est Lacan qui le dit) la relation avec le Cogito, lequel est lui aussi une manière de point géométral, de point de perspective», écrit DAMISCH, ibidem, p. 55.

(23) H. Damisch, L'origine de la perspective, op. cit., p. 122. Il conviendrait d'ajouter à cette référence le livre de G. Didi-Huberman, Ce que nous voyons, ce qui nous regarde, Paris, Éditions de Minuit, 1992 («Critique»). 
prit des spectateurs du théâtre et dans celui des gens du peuple est-elle, dans sa genèse, comparable? Qu'est-ce que penser face à un spectacle, si ce n'est pas être victime de son imagination? Marivaux introduit alors un autre personnage: «Quelqu'un de la bande [...] ne trouvait pas apparemment son compte à fournir son contingent d'étonnement pour le discours de notre politique.» Un dialogue s'engage:

Un petit mot, Monsieur, lui dit-il, de l'air d'un homme qui ne se paie pas de babil et qui a trop d'esprit pour s'épouvanter de celui d'un autre; prenez garde que ces ministres que vous louez tant, auraient pu dans une telle occasion... Monsieur, lui répondit l'autre en lui coupant la parole, je ne force personne et vous êtes libre d'en penser ce qu'il vous plaira; ce que j'ai dit n'en est pas moins juste. Le censeur, à ces mots, sourit d'un air incrédule et se tut.

L'idée d'une opinion publique est-elle enfin concevable, sinon vérifiable? C'est le dialogisme qui permet à Marivaux de l'expérimenter. Il semble à la fois noter l'effet pervers de pompes sur le jugement de celui qui s'identifie aux ministres, et reprendre le censeur oisif qui avance une appréciation ne reposant au final que sur son amour- propre. L'un et l'autre sont certes vaniteux. Mais chez Marivaux, la vanité n'est pas qu'un défaut: il arrive qu'elle soit «subtile», qu'elle «nous éclaire», de même d'ailleurs que la coquetterie ${ }^{24}$. Sans prendre parti sur le fond de la question politique soulevée par ces personnages, il fait quand même dire «vous êtes libre d'en penser ce qu'il vous plaira» à l'un des deux protagonistes, ce qui signale que les gens du peuple - et il n'est plus question de «populace»-savent parfaitement discerner la portée de l'alliance avec l'Espagne, et même laisser la place à une diversité de «points de vue», dût leur vanité en souffrir...

\section{La misanthropie, ou: comment assigner un point de vue sur la vie sociale?}

Pour voir et concevoir, il faut une prise de distance, constituer l'objet de la vue en scène, et s'impliquer dans l'ensemble du dispositif. Marivaux a donc dû adopter un point de vue, au sens à la fois optique et politique. Et c'est encore une profonde réflexion sur des questions centrales posées par Pascal qui l'ont amené, selon nous, à concevoir ce «reportage» en termes de prise de recul, de déplacements successifs de la perspective. «Je ne puis juger d'un ouvrage en le faisant: il faut que je fasse comme les peintres et que je m'en éloigne, mais non pas trop. De combien donc? Devinez.» (Pensées, fragment 465). «Trop de distance ou trop de proximité empêche la vue.» (fragment 230). Ou encore, fragment 99: «Une ville, une campagne, de loin c'est une ville et une campagne, mais à mesure que l'on s'approche ce sont des maisons, des arbres, des tuiles, des feuilles...» Enfin, le fragment 55 «La perspective assigne [le véritable lieu d'où voir un tableau] dans la peinture. Mais dans la vérité et dans la morale, qui l'assignera?». Toutes ces questions taraudent Marivaux dès ses premières œuvres. Dans un article pour Le Mercure de mars 1719, il écrivait:

Il est un certain point de clarté au-delà duquel toute idée perd nécessairement de sa force ou de sa délicatesse. Ce point de clarté est, aux idées, ce qu'est, à certains objets, le point de distance auquel ils doivent être regardés, pour qu'ils offrent leurs beautés attachés à cette distance $e^{25}$.

(24) «Quoi qu'on dise de notre amour-propre, il nous éclaire à merveille...», est-il écrit dans la dix-huitième Feuille du «Spectateur français». Voir aussi les scènes de femme à sa toilette, en particulier dans ses Lettres sur les habitants de Paris, ou encore dans cette même Feuille n. 18, pp. 214-215 de l'édition des Journaux.

(25) Marivaux, Pensées sur différents sujets, dans 
On a pu le constater plus haut: Marivaux prélève des éléments du réel, il «documente», mais ne peut en même temps s'empêcher d'interposer, en écran, du discours, des idées. Ainsi des «enseignes». Il est à la limite entre deux épistémè, l'une de la référence et de l'imitation (d'où l'intertexte pascalien), l'autre de la captation impromptue du réel que l'on trouvera plus tard esquissée chez un Restif (Les nuits de Paris), et de façon plus brute au $\mathrm{xx}^{\mathrm{e}}$ siècle, avec l'influence de la photographie sur la poésie du quotidien (chez Apollinaire ou Cendrars). Il lui faut donc penser son dispositif optique, visuel et idéel, ce qu'il appelle le «point de clarté».

D'où la catégorie du «misanthrope», lequel est paradoxalement la source et le destinataire du spectacle, apuré de sa composante illusionniste. Trait générique de la posture du «journaliste» de l'époque (Van Effen, notamment, en 1711 avait publié, aux Pays-Bas, un journal titré ainsi), le personnage du spectateur misanthrope trouve son origine dans la fiction expliquant l'écriture de ce journal (son auteur, anonyme, étant censé être un homme âgé, quelque peu bougon, retiré du monde et éloigné des passions de ses contemporains). Surtout, la misanthropie est ici une nécessité anthropologique, équivalente à ce que Marivaux dénomme pour le style le «point de clarté». L'ami de la sagesse, le philosophe, ne saurait faire autrement que de prendre ses distances à l'égard des hommes, tant par les conclusions qu'il tire de ses observations que par souci de méthode. Fréquenter les hommes, c'est forcément apprendre qu'ils ne sont ni bons ni vertueux, donc qu'il vaut mieux s'en écarter. Mais les étudier, c'est s'efforcer de les objectiver. Vis-à-vis des hommes, le sage doit régler son écartement, tant pour s'en préserver que pour les observer. Le Spectateur pertinent sera celui qui joue en permanence le pari d'un équilibre entre les deux termes contraires, entre philo- et miso-, aimer et haïr. En réalité, les deux termes en impliquent quatre. Pour être autant misanthrope que philosophe, le Spectateur est forcément un peu aussi philanthrope et «misosophe». Car les hommes, même si l'on doute qu'il soit utile de leur enseigner la sagesse, suscitent malgré tout l'attachement. Et puis, à quoi servirait-il de les moraliser, si c'était pour leur répéter qu'ils sont damnés à tout jamais? Le Spectateur de Marivaux doit par conséquent s'écarter autant des hommes que de la morale qu'on leur enseigne communément et par laquelle on les estime trop ou pas assez. Quant à la philosophie, un personnage de la feuille XXI, publiée en octobre 1723 , viendra lui tordre le cou:

Laissez à certains savants, je veux dire aux faiseurs de systèmes, à ceux que le vulgaire appelle philosophes, laissez-leur entasser méthodiquement visions sur visions en raisonnant sur la nature des deux substances, ou sur choses pareilles.[...] Nous ne sommes pas en ce monde en situation de devenir savants; nous ne sommes encore que l'objet, ou plutôt le sujet, de cette science que nous voudrions avoir ${ }^{26}$.

Remarquons le mot «visions»: il s'agira de substituer à celles des philosophes (au sens de conceptions abstraites) celle, ponctuelle et singulière, d'un Spectateur misanthrope qui ne voit les hommes ni de leur propre point de vue ni du lieu de pouvoir qui met en scène le spectacle. Remarquons aussi, dans la figure de correction allant du mot «objet» à celui de «sujet», que Marivaux énonce on ne saurait plus clairement l'aporie caractérisant tout savoir anthropologique: la confusion inéluctable entre observateur et observé, l'implication de l'expérimentateur dans son expérience. Pour se voir, et en dépit des ambitions et des prétendues méthodes de tous les philosophes, l'homme ne saurait se dissocier de lui-même: «Voilà comment nous raisonnerions, si

Journaux et Euvres diverses, op. cit., p. 54. Nous soulignons.
(26) MarivauX, «Le spectateur français», ibid., p. 232. Nous soulignons. 
nous pouvions nous séparer de nous-mêmes, et nous considérer dans l'homme» ${ }^{27}$. La misanthropie du Spectateur est en réalité un postulat à valeur épistémologique ainsi qu'une rupture avec la posture moraliste traditionnelle: tout n'est qu'une question d'instauration du point de vue et de réglage de la distance, et de mise en perspective d'un point de fuite. Comme le rappelle Hubert Damisch:

La rotation à $180^{\circ}$ du panneau sur lequel était peinte l'image du baptistère [de Brunelleschi] et l'invitation faite au spectateur de placer son œil au revers du trou dont il était percé en son centre représentaient l'équivalent expérimental du mouvement d'objectivation, [...] par laquelle le regard s'arrache à l'opacité du donné pour s'établir en vis-à-vis par rapport à lui. Pour que les choses et le monde deviennent objets pour la perception, le sujet doit prendre recul par rapport à lui-même, n'y ayant de vision ni de connaissance qui ne procède, au plus profond, d'une telle rotation, en même temps que de l'élévation, de l'ostension de l'objet qui en est le corollaire. Mais ce mouvement, jusque dans ce qu'il peut avoir d'un peu théâtral, reste soumis à la loi qui est celle de la représentation: la distance que le sujet prend par rapport à l'objet lui permet d'échapper à l'immédiateté du vécu; mais il ne réussit qu'à s'y découvrir impliqué, irrémédiablement, dans un spectacle qui emprunte sa vérité de cette implication même ${ }^{28}$.

Mais comment trouver ce point de juste vision, dont la pertinence ne saurait être théoriquement décidée, mais, seulement, empiriquement éprouvée? L'on voit bien poindre le problème: qu'est-ce qui va pouvoir garantir de l'arbitraire cette empirie individuelle, et qu'est-ce qui peut valider un regard sur la vie politique, qui ne soit ni celui du peuple ni celui du prince? Le Prince existe-t-il hors du regard du peuple? Et le peuple même est-il une entité unifiée, sans le regard que lui accorde son Prince? N'est-ce pas dans une sorte d'aller-retour entre ces deux regards que se constitue la communauté politique? Là intervient la catégorie sociale et politique de l'amour: effet du va-et-vient en miroir de la relation du peuple au prince, d'une double projection, d'une réciprocité du point de vue et du point de fuite, solidaires dans le dispositif optique que Marivaux imagine servant de fondement à la relation politique, à sa scène, dont le théâtre devient naturellement à la fois le modèle théorique et la garantie empirique. L'amour renvoie aussi à une autre idée pascalienne transformée par Marivaux, celle d'infini, étroitement liée à celle de point de fuite. L'amour projeté par le peuple, alimenté par le prince aimé parce qu'aimant et réciproquement devient le seul sentiment parmi les sentiments politiques (le respect, la crainte, etc.) qui soit potentiellement infini. Cet infini, que Pascal nomme une idée «de derrière la tête» ${ }^{29}$, et qui chez lui sapait tout rapport humain stable, alimente chez Marivaux l'utopie d'un «monde vrai» ${ }^{30}$ reposant sur l'amour, moteur indéfiniment rechargeable d'une relation elle-même perpétuellement renouvelable puisque basée sur un effet optique de miroir: le peuple regardant le prince ne voit que sa propre unité, attestée par le regard du prince, etc. Vision idéale? N'était l'inconstance (parfois «double»!) qui dans la vie sentimentale aussi bien que politique vient compliquer et relancer la règle du jeu...

Le problème essentiel de Marivaux est donc l'établissement d'une perspective légitime sur les comportements des hommes, et inversement (car toute perspective joue symétriquement d'un point de vue et d'un point de fuite) l'adaptation du juge-

(27) Marivaux, «Le Cabinet du philosophe», ibid., p 354 .

(28) H. Damisch, L'origine de la perspective, op. cit., p. 345 .

(29) Sur l'infini comme «pensée de derrière la tête», voir L. MARIN, Critique du discours, Paris, Édi- tions de Minuit («Critique»), 1975, pp. 395-400.

(30) Sur la suite de voyages dans le «Monde vrai», voir «Le cabinet du philosophe», dans MARIVAUX, Journaux et Euvres diverses, op. cit., feuilles VI à XI. 
ment moral (au double sens de législation et d'appréciation) à ce qu'il en est d'eux. Marivaux s'ancre résolument dans l'empirie après avoir écarté la solution pascalienne d'adopter un point de vue «fou», celui de l'infini, le seul satisfaisant en termes d'absolu, mais qui, bien entendu, est à la fois impossible et signe justement de la finitude de la raison humaine.

Car «légitime» signifie d'abord humaine. Nul ne saurait adopter un point de vue divin sur les hommes; quand ce serait possible, ce serait une usurpation. La légitimité des vues morales est affaire de droit autant que de conditions matérielles d'observation. Au contraire de ses prédécesseurs, Marivaux ne va donc pas décrire des «types», des «caractères» abstraits. Il ne voit ni depuis les coulisses, ni de la loge royale. Son Spectateur n'est qu'un quidam, il fait lui-même, in posse, partie du spectacle que les hommes donnent en permanence, à leur corps défendant. Mais justement, la question centrale va être pour lui celle du «corps politique». Qui ne saurait faire abstraction, en dépit de sa généralité même, de celle des corps concrets, ceux des hommes et des femmes qui figurent dans la «salle» du spectacle sans être justement de simples figurants. Il se met en disposition de rencontrer des cas d'bumanité (au sens où la tradition moraliste parlait de casus: motif de réflexion sur les comportements; mais ici, sans qu'il y ait de préoccupation normative) là où ils sont, y compris dans la foule. Rechercher l'homme au milieu des autres, c'est respecter sa grégarité de fait, instituer le droit pour un parmi tous de les observer chacun en particulier, quitte à connaître des difficultés pour isoler les «cas» le temps de l'examen. Aussi bien, la foule, loin de disséminer les hommes, de rendre impossible un point de vue global sur eux, les concentre et les révèle aux yeux de celui qui ne prétend à rien, sinon à être leur semblable. Car le Spectateur ne jouit d'aucun privilège sur ceux qu'il observe. Cela lui permet, dans la suite, de s'adresser solennellement aux Rois, qui bénéficient de prérogatives exceptionnelles (dont celle d'être «considérés» par les hommes qui les voient passer), quoique légitimes, au moins tant qu'ils ne les confondent pas avec des privilèges, justement ${ }^{31}$.

La collectivité est donc la dimension primordiale sinon principielle de l'homme. L'on est bien avant Rousseau, et l'homme «au naturel» n'est jamais seul, selon Marivaux: la foule condense son essence, et «la science du cœur humain» telle qu'on peut l'acquérir «réside dans le commerce que nous avons tous, et sans exception, ensemble» ${ }^{32}$. Le Spectateur, anonyme et neutre, est là pour la considérer et la comprendre (c'est tout un), sans que sa position implique qu'il s'adjuge à lui-même quelque supériorité que ce soit. Ce dont il avertissait dès le début de la feuille V:

Cette innombrable quantité d'espèces de mouvements forme à ses yeux un caractère générique. À la fin, tant de sujets se réduisent en un; ce n'est plus des hommes différents qu'il contemple, c'est l'homme représenté dans plusieurs mille".

La rue est à la fois ce lieu public où il arrive que le «journaliste» rencontre des événements et le «théâtre» où le Spectateur se réjouit de faire son entrée, car elle est l'espace même du politique, sa «scène», dirait Jacques Rancière ${ }^{34}$. Si c'est le cas, le lieu de réalisation autant que de légitimation de ce regard ne pourra se formuler que par l'invention d'un concept neuf, celui d'opinion publique. Tel sera le fruit de la «récolte d'idées».

(31) Cette distinction essentielle entre privilège et prérogative se retrouve, en 1723, dans La double inconstance, lors des confrontations d'Arlequin avec le Seigneur (III, 4).

(32) Marivaux, Réflexions sur l'esprit bumain, dans Journaux et Euvres diverses, op. cit., p. 476.

(33) Marivaux, «Le spectateur français», ibid., p. 132-33.

(34) Voir Le partage du sensible, La Fabrique, 2000. 
Car si l'expression n'est pas chez Marivaux, tout atteste ici qu'il en a pensé sinon l'effectivité, du moins la possibilité. Évidemment, il ne s'agit pas de proposer une date à partir de laquelle le peuple (ou quiconque d'ailleurs) commencerait à «se faire des idées» sur son entourage social: commenter des nouvelles, savoir aussi qu'il faut se défier des «mouches» qui espionnent, diffuser des rumeurs ou des libelles pour influer sur la renommée bonne ou mauvaise de tel parti ou personnage, tout cela existe de l'Antiquité au Moyen Age, c'est assez clair ${ }^{35}$. Il ne l'est pas moins qu'un ouvrage tel que les Provinciales de Pascal, outre qu'il est un admirable instrument de propagande, contient aussi une théorie de la communication, reposant sur le partage fait entre raison et imagination, renvoyant à l'anthropologie de son auteur. Néanmoins, nombreux sont ceux qui, en effet, ont remarqué qu'au XVIIIe siècle s'était opérée une mutation qualitative, faisant que l'on était passé d'une opinion répandue dans le public à une réalité nouvelle, la moderne «opinion publique».

L'on sait que Rousseau datait du milieu du siècle l'apparition du phénomène, tandis que Habermas gratifiait les physiocrates d'avoir, les premiers, pensé son rôle politique $^{36}$. La plupart des historiens s'accordent pour remarquer que la notion de «public opinion» est enregistrée en Angleterre dès les années 1730, tandis qu'en France, en raison du cloisonnement des institutions et des circuits du commerce intellectuel, ce concept demeure flou, voire dénié. Ainsi, à la fin du siècle Saint-Just préfèret-il «conscience publique» ou «esprit public», termes davantage chargés d'éthique, opinion étant classiquement opposé à la connaissance vraie ${ }^{37}$. Si Malesherbes faisait remonter la naissance de l'opinion publique à l'institution de l'Académie française, c'était pour accentuer l'importance des gens de lettres dans l'éclairement des esprits. Manière de souligner que le peuple ne saurait par lui-même pourvoir à ses propres lumières. Telle sera encore l'appréciation de Condorcet, écrivant en 1776:

Quand on parle d'opinion, il faut en distinguer trois espèces: l'opinion des gens éclairés, qui précède l'opinion publique et finit par lui faire la loi; l'opinion dont l'autorité entrâne l'opinion du peuple; l'opinion populaire enfin, qui reste celle de la partie du peuple la plus stupide et la plus misérable ${ }^{38}$.

Nous sommes ici, avec Marivaux, au moins vingt-cinq ans avant la date posée en jalon par Jean-Jacques, quarante ans avant les réflexions de Turgot; à bien y regarder, la conception qu'il se fait de la formation et la légitimité de l'opinion est moins rigide que celle de Saint Just (dieu merci!), plus moderne même que celle de Condorcet!

C'est qu'ici Marivaux, parce qu'il est à la fois sur les deux terrains du théâtre et de la relation au public contenue dans sa notion du journalisme ${ }^{39}$, non seulement

(35) «Jacques Le Goff le souligne, le XIII ${ }^{\mathrm{e}}$ siècle est celui où naît l'opinion publique», rappelle Denis Hüe dans son ouvrage sur le poète Rutebeuf, à l'époque duquel l'on a de bons exemples, déjà, de captation de l'opinion, grâce à des textes écrits ou oraux (les libelli famosi) dans des milieux populaires ou estudiantins. Voir J. LE GoFf, Saint Louis, Paris, Gallimard, 1996, p. 104. Voir aussi D. HüE, Rutebeuf, Paris, Atlande, 2006, p. 42.

(36) «Parmi les singularités qui distinguent le siècle où nous vivons de tous les autres est l'esprit méthodique et conséquent qui dirige depuis vingt ans les opinions publiques», écrivait-il dans Rousseau juge de Jean-Jacques en 1776. Voir J. HABERMAS, L'Espace public. Archéologie de la publicité comme dimension constitutive de la société bourgeoise, $\mathrm{Pa}$ - ris, Payot, 1978.

(37) SAInT-Just, Rapport à la Convention du 28 germinal an II, cité par M. Ozouf dans «Le concept d'opinion publique au XVIII siècle», article publié dans L'bomme régénéré, Essais sur la Révolution française, Paris, Gallimard, 1989 («Bibliothèque des histoires»), p. 53.

(38) Condorcet, Réflexions sur le commerce des blés, Londres, 1776, p. 140. Cité par M. Ozouf, op. cit., p. 33.

(39) Voir les feuilles IX et XII du Spectateur français, aux pages 156 et 175 de notre édition, les lettres supposément adressées à M. le Spectateur par une lectrice ou un lecteur. Même si Marivaux ne dispose pas de bureau d'adresse véritable pour le courrier des lecteurs, on le voit nettement 
enregistre la possibilité théorique de la formation de l'opinion publique, mais encore valide moralement son existence et en atteste la légitimité politique. Il n'est pas dans une dichotomie opposant la conscience politique à la fascination servile. Il constate l'une et l'autre, non exclusives, liées l'une à l'autre au contraire. Marivaux ne note pas que les gens qu'il croise ont des opinions, saines ou confuses, il fait autre chose.

Ainsi Brunelleschi avait-il pensé le fait perspectif, la relation de l'œil à l'espace, non par une vue directe mais en construisant un dispositif en miroir lui permettant de réfléchir, mesurer et cadrer. Marivaux également dispose son champ optique à $180^{\circ}$, dos à l'événement, afin de concevoir une raison des effets qui s'y produisent. Telle est l'opinion publique moderne: non une collection de sentiments singuliers, mais bel et bien une structure en miroir (un espace de jeu et de regards entre le pouvoir et ses «administrés») informant le mental de la société tout entière. Elle est, entre gouvernants et gouvernés, un contrat social imaginaire, tangible pourtant comme l'est une passion éprouvée par une foule, reconductible mais pas inconditionnelle. Marivaux, semble-t-il, perçoit le phénomène dans sa complexité. Elle consiste en l'articulation de la masse opinant et des individus qui, la formant, n'en présentent pas moins des différences, voire des contradictions les uns avec les autres. L'opinion publique n'est pas davantage la masse unanime qu'une collection confuse d'avis inconciliables engendrant (ce sera la crainte de Rousseau) l'inimitié entre tous. Penser l'opinion publique moderne, ce sera concevoir à la fois les divergences et l'unité. Comme le souligne très clairement Mona Ozouf:

Il n'y aurait pas d'opinion publique s'il n'y avait pas d'opinion individuelle, c'est-à-dire des êtres indépendants capables d'opiner. On peut même suggérer que l'opinion publique n'a cette force d'invocation que parce que le lien social n'est plus senti comme premier et que son effacement a découvert les opinions particulières, logiquement antérieures, logiquement indépendantes et logiquement égales entre elles ${ }^{40}$.

Contrairement à ce que penseront certains physiocrates ou révolutionnaires, les questions «faut-il des formateurs d'opinion pour éclairer le peuple?», «faut-il attendre que le peuple soit éclairé pour songer à un suffrage universel?» ne se posent pas, dès lors que l'on est comme Marivaux convaincu qu'il n'y a «point d'homme qui n'ait sa part universelle d'intelligence et de capacité, autrement dit son aptitude générale pour tout ce qui peut occuper et exercer l'esprit humain ${ }^{41}$. C'est ce qui lui permet, avec cette force et cette légèreté particulières à la feuille $\mathrm{V} \mathrm{du}$ «Spectateur français», de constater la disparité des opinions, leur validité relative, et la compatibilité de leur coexistence au sein d'un peuple réuni et divers, dont le lien social qui l'unit au prince repose moins en effet sur la fascination que sur l'idée en germe de contrat, au double sens politique et amoureux.

JEAN-PIERRE MONTIER

sensible aux effets de retour propres à la relation journalistique. Celle-ci, à la différence de la relation littéraire, ne s'oriente pas en direction de milieux sociaux nettement délimités, et conçoit un lectorat aux opinions duquel le rédacteur se doit d'être im- médiatement réactif.

(40) M. Ozouf, L’homme régénéré, Essais sur la Révolution française, cit., p. 40.

(41) Marivaux, Réflexions sur l'esprit bumain, dans Journaux et Euvres diverses, cit., p. 484. 